\title{
Google revives controversial cold-fusion experiments
}

\section{Researchers found no evidence for the phenomenon, first proposed 30 years ago.}

\section{BY ELIZABETH GIBNEY}

S ince 2015, Google has been funding experiments into cold fusion - the theory that nuclear fusion, the process that powers the Sun, can produce energy in a table-top experiment. Two scientists first made sensational claims about achieving the phenomenon promising endless, cheap energy - 30 years ago, but their results were quickly debunked and the topic is now considered a scientific taboo.

Google's project - revealed this week in Nature - found no evidence that cold fusion is possible, but made some advances in measurement and materials-science techniques that the researchers say could benefit energy research (C. P. Berliguette et al. Nature http://doi.org/ c6dh; 2019). The team also hopes that its work will inspire others to revisit cold-fusion experiments, even if the phenomenon still fails to materialize.

"This is not just a chase for cold fusion," says Matthew Trevithick, a research programme manager at Google in Mountain View, California. "If it were, I don't think we would have maintained an interest of this calibre of team for so long."

The Google team explored three experimental set-ups involving metals and hydrogen that have been proposed to generate cold fusion. Their results have been published across 12 papers over the past 2 years.
Some scientists welcomed the scrutiny brought by the Google project. But Frank Close, a theoretical physicist at the University of Oxford, UK, says that the scientific mainstream has shunned the topic for good reason: no one has managed to independently reproduce the finding and more worthwhile topics have emerged, he says.

\section{NOTORIOUS CLAIMS}

In March 1989, two US-based chemists, Stanley Pons and Martin Fleischmann, announced that they had seen signs of nuclear fusion when they ran a current across two palladium plates in water laden with deuterium, a heavy isotope of hydrogen. Others quickly pointed out errors in their experimental procedure and no experiment has ever reproduced the result. But cold fusion - now commonly referred to as low-energy nuclear reactions - has retained a persistent following that continues to claim evidence of success.

Google's US\$10-million project aimed to test the cold-fusion claims rigorously, says Trevithick.

Nuclear fusion is thought to happen only in extreme environments such as the Sun, where high temperatures and pressures cause hydrogen atoms to overcome their mutual repulsion and fuse into helium, releasing enormous amounts of energy. The probability of atoms fusing at much lower temperatures is thought

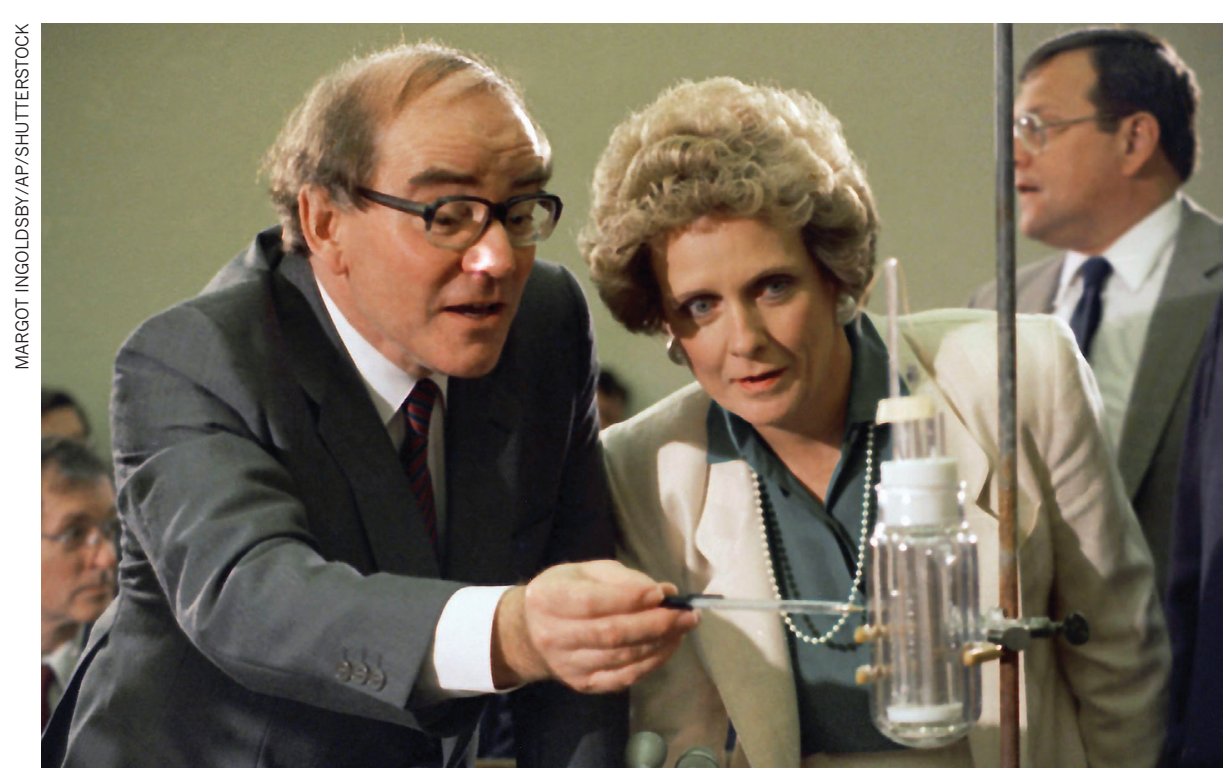

Chemist Martin Fleischmann (left) was part of a team that claimed to have produced cold fusion in 1989. to be vanishingly small. But, if it were possible, this phenomenon would bring enormous energy benefits.

Google's team of 30 researchers pursued three experimental strands linked to cold fusion. In one, they tried to load palladium with amounts of deuterium suggested to be necessary to trigger fusion. But at high concentrations, the team was unable to create stable samples. A second strand followed up on 1990s work by US physicists who claimed to have generated anomalous levels of tritium another heavy hydrogen isotope, created only through nuclear reactions - by bombarding palladium with pulses of hot deuterium ions. The Google team found no tritium production in this set-up.

A final strand heated metallic powders in a hydrogen-rich environment. Some current proponents of cold fusion claim that the process produces excess and unexplained heat, which they theorize is the result of fusing elements. But across 420 tests, the team found no such heat excess.

However, the researchers say that both palladium experiments warrant further study. The proposed effects in the tritium experiment could be too small to measure with current equipment, they say. The team also says that further work could produce stable samples at extremely high deuterium concentrations.

All the projects pushed the frontier of experimental methods, says Trevithick, including developing "the best calorimeters in the world" to detect even slight excesses of heat under extreme experimental conditions. These could potentially be used to test future claims.

\section{PUSHING THE ENVELOPE}

"I think that the authors have done a really good job," says David Williams, an electrochemist at the University of Auckland in New Zealand - especially in how they have navigated the controversial topic. Pushing the envelope of measurement science is also important, says Williams, whose team did some of the first failed replication studies of the original claim.

But Close, who was involved in early replication attempts, says that being unable to rule an idea out completely does not mean there is good reason to pursue it. "If somebody I was investing my money in started doing this, I would withdraw my money," says Close. 